\title{
DIVIDED DIFFERENCES AND SYSTEMS OF NONHARMONIC FOURIER SERIES ${ }^{1}$
}

\author{
DAVID ULLRICH
}

\begin{abstract}
Suppose that $\omega_{n, 0}, \omega_{n, 1}, \ldots, \omega_{n, k}$ are distinct complex numbers with $\left|n-\omega_{n, j}\right|<\delta$ for all $n \in \mathbf{Z}, j=0,1, \ldots, k$. We show that if $\delta>0$ is small enough then, given complex numbers $c_{n, j}(n \in Z, j=0,1, \ldots, k)$ there exists $f \in L^{2}(-(k+1) \pi,(k+1) \pi)$ with

$$
\int_{-(k+1) \pi}^{(k+1) \pi} f(t) e^{-i t \omega_{n j}} d t=c_{n j} \text { for } n \in \mathbf{Z}, j=0,1, \ldots, k
$$

if and only if certain "divided differences" involving the $c_{n j}$ 's and the $\omega_{n j}$ 's are square summable. This extends a classical theorem of Paley and Wiener, which is equivalent to the case $k=0$ above.
\end{abstract}

0. Introduction. A classical theorem of Paley and Wiener [5, p. 113] states that if $\delta<1 / \pi^{2}$ and $\left\{\omega_{n}\right\}$ is a sequence of reals satisfying $\left|n-\omega_{n}\right|<\delta$ for $n \in \mathbf{Z}$, then the functions $e^{i \omega_{n} t}$ form a "Riesz basis" for $L^{2}[-\pi, \pi]$. This conclusion is equivalent to the following statement: Suppose $\left\{c_{n}\right\}(n \in \mathbf{Z})$ is a sequence of complex numbers. Then the interpolation problem

$$
\int_{-\pi}^{\pi} f(t) e^{-i \omega_{n} t} d t=c_{n} \quad(n \in \mathbf{Z})
$$

has a solution $f \in L^{2}[-\pi, \pi]$ if and only if $\sum_{-\infty}^{\infty}\left|c_{n}\right|^{2}<\infty$. (Kadec [3] has shown that this holds under the weaker hypothesis $\delta<\frac{1}{4}$, which is known to be the best possible result; see [4, p. 48].)

Young [8] has recently established an interesting generalization of this result; the purpose of the present paper is to give another generalization, of an entirely different sort. Consider the following problem: Suppose $K$ is a positive integer, and the $\omega_{n, j}(n \in \mathbf{Z}, 0 \leqslant j \leqslant K)$ are complex numbers with each $\omega_{n, j}$ "close enough" to $n$. For what choices of complex numbers $c_{n, j}$ will the system

$$
\int_{-(K+1) \pi}^{(K+1) \pi} \leqslant(t) e^{-i \omega_{n j} t}=c_{n, j} \quad(n \in \mathbf{Z}, 0 \leqslant j \leqslant K)
$$

have a solution $f \in L^{2}[-(K+1) \pi,(K+1) \pi]$ ? In Theorem 3 below we demonstrate the existence of $\delta=\delta_{K}>0$ such that under the hypothesis $\left|n-\omega_{n, j}\right|<\delta_{K}$ ( $n \in \mathbf{Z}, 0 \leqslant j \leqslant K$ ), the system (1) has a solution $f \in L^{2}[-(K+1) \pi,(K+1) \pi]$ if and only if certain "divided differences" involving the $c_{n, j}$ are square summable. (See Theorem 3 for the precise statement.)

Received by the editors May 10, 1977 and, in revised form, April 4, 1979.

AMS (MOS) subject classifications (1970). Primary 42A80, 42A12; Secondary 42A84.

Key words and phrases. Nonharmonic Fourier series, Riesz basis, divided difference.

${ }^{1}$ The writing of an earlier version of this paper was supported by a grant from the University of Wisconsin Honors Program. 
[Note. Throughout this paper "scalar" means "complex number", "Hilbert space" means "complex Hilbert space", etc.]

\section{Riesz bases.}

Definitions. Let $\left\{x_{n}\right\}$ and $\left\{y_{n}\right\}(n \in \mathbf{Z})$ be sequences of elements of the Hilbert space $H$. Then $\left\{y_{n}\right\}$ is biorthogonal to $\left\{x_{n}\right\}$ if for $n, m \in \mathbf{Z}$

$$
\left\langle x_{n}, y_{m}\right\rangle=\delta_{n, m}= \begin{cases}1 & \text { if } n=m, \\ 0 & \text { if } n \neq m .\end{cases}
$$

The sequence $\left\{x_{n}\right\}$ is independent if there exists a sequence $\left\{y_{n}\right\}$ which is biorthogonal to $\left\{x_{n}\right\}$. The sequence $\left\{x_{n}\right\}$ is complete in $H$ if 0 is the only element of $H$ which is orthogonal to each $x_{n}(n \in \mathbf{Z})$. (Equivalently, $\left\{x_{n}\right\}$ is complete if and only if its linear span is dense in $H$.)

The following theorem is essentially a summary of various "well-known" facts, many of which are contained, at least implicitly, in [5, Chapter VII], and in [1]. It and the lemma which follows it are included here primarily for the convenience of the reader.

TheOREM 1. Let $\left\{x_{n}\right\}(n \in \mathbf{Z})$ be a sequence of elements of the Hilbert space $H$. The following are equivalent:

(i) For scalars $c_{n}(n \in \mathbf{Z})$ the system $\left\langle y, x_{n}\right\rangle=c_{n}(n \in \mathbf{Z})$ has a solution $y \in H$ if and only if $\sum_{-\infty}^{\infty}\left|c_{n}\right|^{2}<\infty$.

(ii) There exist $A, B>0$ such that for any $N$ and for any scalars $a_{-N}, \ldots, a_{N}$,

$$
A^{2} \sum_{-N}^{N}\left|a_{n}\right|^{2} \leqslant\left\|\sum_{-N}^{N} a_{n} x_{n}\right\|^{2} \leqslant B^{2} \sum_{-N}^{N}\left|a_{n}\right|^{2} .
$$

Further, if $\left\{x_{n}\right\}$ is complete in $H$, then (i) and (ii) are each equivalent to each of the following:

(iii) There exists $\left\{y_{n}\right\}$ biorthogonal to $\left\{x_{n}\right\}$, with $\left\{y_{n}\right\}$ complete in $H$, and there exist $A, B>0$ such that for any $N$ and for any scalars $c_{-N}, \ldots, c_{N}$

$$
B^{-2} \sum_{-N}^{N}\left|c_{n}\right|^{2} \leqslant\left\|\sum_{-N}^{N} c_{n} y_{n}\right\|^{2} \leqslant A^{-2} \sum_{-N}^{N}\left|c_{n}\right|^{2} .
$$

(iv) For any scalars $a_{n}(n \in \mathbf{Z})$ such that $\sum_{-\infty}^{\infty}\left|a_{n}\right|^{2}<\infty, \quad \sum_{-\infty}^{\infty} a_{n} x_{n}=$ $\lim _{N \rightarrow \infty} \Sigma_{-N}^{N} a_{n} x_{n}$ exists in $H$, and for any $x \in H$ there exists a unique sequence $\left\{a_{n}\right\}$ with $\sum_{-\infty}^{\infty}\left|a_{n}\right|^{2}<\infty$ and $x=\sum_{-\infty}^{\infty} a_{n} x_{n}$.

[Note. The limit in (iv) refers to the norm topology on $H$.]

Proof. Suppose, to start with, that $\left\{x_{n}\right\}$ is complete in $H$. We show that (i) $\Rightarrow$ (iii) $\Rightarrow$ (ii) $\Rightarrow$ (iv) $\Rightarrow$ (i).

Let $l^{2}=l^{2}(Z)$ be the Hilbert space of square summable sequences, indexed by $\mathbf{Z}$; for any $\zeta \in l^{2}$ and $n \in \mathbf{Z}$, we will denote by $\zeta_{n}$ the $n$th component of $\zeta$; $e_{n}$ will be the element of $l^{2}$ with $\left(e_{n}\right)_{m}=\delta_{n, m}(n, m \in \mathbf{Z})$.

(i) $\Rightarrow$ (iii): Suppose (i). This says precisely that we can define a linear map $T$ : $H \rightarrow l^{2}$ by

$$
(T x)_{n}=\left\langle x, x_{n}\right\rangle
$$


and that $T(H)=l^{2}$. The closed graph theorem implies that $T$ is bounded, and the completeness of $\left\{x_{n}\right\}$ shows that $T$ is one-to-one. Hence (by the open mapping theorem) $T^{-1}$ exists and is bounded. Let $y_{n}=T^{-1}\left(e_{n}\right)$. Then

$$
\left\langle y_{n}, x_{m}\right\rangle=\left(T y_{n}\right)_{m}=\left(e_{n}\right)_{m}=\delta_{n, m},
$$

so $\left\{y_{n}\right\}$ is biorthogonal to $\left\{x_{n}\right\}$. Since $T$ is a Banach space isomorphism, the completeness of $\left\{e_{n}\right\}$ in $l^{2}$ gives completeness of $\left\{x_{n}\right\}$ in $H$. (See the comment after the definition of "complete".) Similarly, the two inequalities in (iii) are precisely the statement that $T$ and $T^{-1}$ are bounded.

(iii) $\Rightarrow$ (ii): Suppose (iii). Then we can define a (unique) bounded invertible linear map $T: H \rightarrow l^{2}$ by $T y_{n}=e_{n}$. But now the Hilbert-space adjoint $T^{*}: l^{2} \rightarrow H$ is also invertible, since $\left(T^{*}\right)^{-1}=\left(T^{-1}\right)^{*}$. Since, by (iii),

$$
\left\langle T^{*} e_{n}, y_{m}\right\rangle=\left\langle e_{n}, T y_{m}\right\rangle=\left\langle e_{n}, e_{m}\right\rangle=\delta_{n, m}=\left\langle x_{n}, y_{m}\right\rangle,
$$

the completeness of $\left\{y_{n}\right\}$ shows that $T^{*} e_{n}=x_{n}$. Hence (as above) boundedness of $T^{*}$ and $\left(T^{*}\right)^{-1}$ gives (ii).

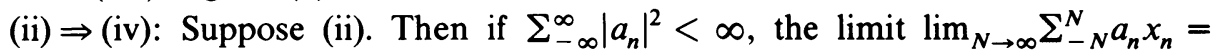
$\sum_{-\infty}^{\infty} a_{n} x_{n}$ certainly exists, by the completeness of $H$. So we may define a linear map $T: l^{2} \rightarrow H$ by $T a=\sum_{-\infty}^{\infty} a_{n} x_{n}$. Then $T$ is bounded and bounded below, by (ii). Since $\left\{x_{n}\right\}$ is complete in $H, T\left(l^{2}\right)$ is dense in $H$; since $T$ is bounded below, $T\left(l^{2}\right)$ is closed. Thus $T\left(l^{2}\right)=H$; similarly $T$ is one-to-one, being bounded below on a dense subspace of $l^{2}$; this gives (iv).

(iv) $\Rightarrow$ (i): Suppose (iv). For each positive integer $N$, define $T_{N}: l^{2} \rightarrow H$ by $T_{N} a=\sum_{-N}^{N} a_{n} x_{n}$. Since

$$
\left\|T_{N} a\right\| \leqslant \sum_{-N}^{N}\left|a_{n}\right|\left\|x_{n}\right\| \leqslant\left(\sum_{-N}^{N}\left\|x_{n}\right\|^{2}\right)^{1 / 2}\|a\|,
$$

each $T_{N}$ is bounded. The hypothesis says that for each $a \in l^{2}, T a=\lim _{N \rightarrow \infty} T_{N} a$ exists in $H$. Hence, by the Banach-Steinhaus theorem, $\left\{\left\|T_{N}\right\|\right\}$ is bounded, and thus $T$ is bounded; the hypothesis also implies that $T$ is one-to-one and onto. Hence, as above, $T$ is invertible, so that $T^{*}$ is also invertible; in particular $T^{*}(H)=l^{2}$. But $\left(T^{*} y\right)_{n}=\left\langle T^{*} y, e_{n}\right\rangle=\left\langle y, T e_{n}\right\rangle=\left\langle y, x_{n}\right\rangle$, so $T^{*}(H)=l^{2}$ gives (i).

Thus far we have proved Theorem 1 in the case that $\left\{x_{n}\right\}$ is complete in $H$. The equivalence of (i) and (ii) in general follows from this case:

Let $H^{\prime}$ be the smallest closed subspace of $H$ containing each $x_{n}(n \in \mathbf{Z})$. Then $\left\{x_{n}\right\}$ is certainly complete in $H^{\prime}$, so we have shown that (i) and (ii) are equivalent if $H^{\prime}$ is substituted for $H$. But the statement of (ii) is entirely unaffected by substituting $H^{\prime}$ for $H$, and (i) is not essentially affected: Let $P: H \rightarrow H^{\prime}$ be the orthogonal projection with $P H=H^{\prime}$; then for $y \in H, n \in \mathbf{Z}$ we have $\left\langle y, x_{n}\right\rangle=\left\langle P y, x_{n}\right\rangle$. Hence for a given sequence of scalars $\left\{c_{n}\right\}$, there exists $y \in H$ with $\left\langle y, x_{n}\right\rangle=c_{n}$ $(n \in \mathbf{Z})$ if and only if there exists $y \in H^{\prime}$ with $\left\langle y, x_{n}\right\rangle=c_{n}(n \in \mathbf{Z})$. Thus the equivalence of (i) and (ii) follows from their equivalence with $H$ replaced by $H^{\prime}$. Q.E.D. 
Definition. Let $\left\{x_{n}\right\}$ be a sequence of elements of the Hilbert space $H$. Then $\left\{x_{n}\right\}$ is a Riesz basis for $H$ if

(i) $\sum_{-\infty}^{\infty} a_{n} x_{n}=\lim _{N \rightarrow \infty} \sum_{-N}^{N} a_{n} x_{n}$ exists in $H$ for any sequence of scalars $\left\{a_{n}\right\}$ satisfying $\sum_{-\infty}^{\infty}\left|a_{n}\right|^{2}<\infty$, and

(ii) for any $x \in H$ there exists a unique sequence of scalars $\left\{a_{n}\right\}$ with $\sum_{-\infty}^{\infty}\left|a_{n}\right|^{2}<$ $\infty$ and $x=\sum_{-\infty}^{\infty} a_{n} x_{n}$.

Thus a Riesz basis is in fact a (Schauder) basis for $H$; Theorem 1 shows that if $\left\{x_{n}\right\}$ is a Riesz basis for $H$, then so is its biorthogonal set. (Note that in general a complete set of vectors has at most one biorthogonal set.)

The proof of the next lemma is essentially the same as that in [6, p. 208], although the statement is more general than that which occurs in [6]. (See also [5, p. 100].)

Lemma 1. Suppose that $\left\{x_{n}\right\}$ is a Riesz basis for the Hilbert space $H$, and $A, B>0$ are chosen so that for any $N$ and for any scalars $a_{-N}, \ldots, a_{N}$

$$
A^{2} \sum_{-N}^{N}\left|a_{n}\right|^{2} \leqslant\left\|\sum_{-N}^{N} a_{n} x_{n}\right\|^{2} \leqslant B^{2} \sum_{-N}^{N}\left|a_{n}\right|^{2} .
$$

(The existence of such $A$ and $B$ is guaranteed by Theorem 1.) Suppose further that $0<A^{\prime}<A$ and $\left\{y_{n}\right\}$ is a sequence of elements of $H$ such that for any $N$ and for any scalars $a_{-N}, \ldots, a_{N}$

$$
\left\|\sum_{-N}^{N} a_{n} y_{n}\right\|^{2} \leqslant\left(A^{\prime}\right)^{2} \sum_{-N}^{N}\left|a_{n}\right|^{2} .
$$

Then $\left\{x_{n}+y_{n}\right\}$ is complete in $H$ and for any $N$ and for any scalars $a_{-N}, \ldots, a_{N}$,

$$
\left(A-A^{\prime}\right)^{2} \sum_{-N}^{N}\left|a_{n}\right|^{2} \leqslant\left\|\sum_{-N}^{N} a_{n}\left(x_{n}+y_{n}\right)\right\|^{2} \leqslant\left(B+A^{\prime}\right)^{2} \sum_{-N}^{N}\left|a_{n}\right|^{2} .
$$

(In particular $\left\{x_{n}+y_{n}\right\}$ is also a Riesz basis for $H$.)

Proof. The inequalities in the conclusion are clear:

$$
\begin{aligned}
\left(A-A^{\prime}\right)\left(\sum_{-N}^{N}\left|a_{n}\right|^{2}\right)^{1 / 2} & \leqslant\left\|\sum_{-N}^{N} a_{n} x_{n}\right\|-\left\|\sum_{-N}^{N} a_{n} y_{n}\right\| \leqslant\left\|\sum_{-N}^{N} a_{n}\left(x_{n}+y_{n}\right)\right\| \\
& \leqslant\left\|\sum_{-N}^{N} a_{n} x_{n}\right\|+\left\|\sum_{-N}^{N} a_{n} y_{n}\right\| \leqslant\left(B+A^{\prime}\right)\left(\sum_{-N}^{N}\left|a_{n}\right|^{2}\right)^{1 / 2} .
\end{aligned}
$$

Now define $T\left(\sum_{-N}^{N} a_{n} x_{n}\right)=\sum_{-N}^{N} a_{n}\left(x_{n}+y_{n}\right)$.

Since

$$
\begin{aligned}
\left\|\sum_{-N}^{N} a_{n}\left(x_{n}+y_{n}\right)\right\| & \leqslant\left\|\sum_{-N}^{N} a_{n} x_{n}\right\|+\left\|\sum_{-N}^{N} a_{n} y_{n}\right\| \\
& \leqslant\left\|\sum_{-N}^{N} a_{n} x_{n}\right\|+A^{\prime}\left(\sum_{-N}^{N}\left|a_{n}\right|^{2}\right)^{1 / 2} \\
& \leqslant\left(1+\frac{A^{\prime}}{A}\right)\left\|\sum_{-N}^{N} a_{n} x_{n}\right\|,
\end{aligned}
$$


we can extend $T$ to a (unique, by the completeness of $\left\{x_{n}\right\}$ ) bounded linear $T$ : $H \rightarrow H$. Let $I$ denote the identity operator; then

$$
\begin{aligned}
\left\|(I-T) \sum_{-N}^{N} a_{n} x_{n}\right\| & =\left\|\sum_{-N}^{N}\left(-a_{n} y_{n}\right)\right\| \\
& \leqslant A^{\prime}\left(\sum_{-N}^{N}\left|a_{n}\right|^{2}\right)^{1 / 2} \leqslant \frac{A^{\prime}}{A}\left\|\sum_{-N}^{N} a_{n} x_{n}\right\| .
\end{aligned}
$$

That is, $\|I-T\| \leqslant A^{\prime} / A<1$, so that $T$ is invertible. I.e., $T$ is a Banach space isomorphism, so the completeness of $\left\{x_{n}\right\}$ and the fact that $T x_{n}=x_{n}+y_{n}$ show that $\left\{x_{n}+y_{n}\right\}$ is complete in $H$. Q.E.D.

\section{Exponentials.}

Notation. If $f$ is a function in $L^{2}(\mathbf{R})$ with compact support and $z \in \mathbf{C}$ we define

$$
\hat{f}(z)=(2 \pi)^{-1 / 2} \int f(t) e^{-i z t} d t
$$

For $r \geqslant 0$ let $I_{r}$ denote the interval $(-(r+1) \pi,(r+1) \pi)$.

The following proposition is quite trivial; we insert it here simply because it would seem awkward to incorporate its proof into that of Theorem 2.

Proposition. If $r \geqslant 0, f \in L^{2}\left(I_{r}\right)$ and $n$ is a nonnegative integer, then there exists $g \in L^{2}\left(I_{r+n}\right)$ with

$$
\hat{g}(z)=(\sin \pi z)^{n} \hat{f}(z) \quad(z \in \mathbf{C}) .
$$

Proof. We need only do the case $n=1$; the general case then follows by iteration. But let $g(x)=(2 i)^{-1}(f(x-\pi)-f(x+\pi))$; then clearly $f \in L^{2}\left(I_{r}\right)$ implies that $g \in L^{2}\left(I_{r+1}\right)$, and one easily verifies that $\hat{g}(z)=\sin (z) \hat{f}(z)$. Q.E.D.

THEOREM 2. If $K$ is a nonnegative integer then the functions

$$
\left\{e^{i n t}, t e^{i n t}, \ldots, t^{K} e^{i n t}\right\} \quad(n \in \mathbf{Z})
$$

form a Riesz basis for $L^{2}\left(I_{K}\right)$.

Proof. First we show completeness. Theorem III in [4] states, in part, that if $\left\{\lambda_{n}\right\}$ ( $n \in \mathbf{Z}$ ) is a sequence of distinct reals, $\Lambda(r)$ is the number of $n \in \mathbf{Z}$ with $\left|\lambda_{n}\right|<r$, and there exists a constant $C$ so that

$$
\int_{1}^{R} \frac{\Lambda(r)}{r} d r \geqslant 2 R-\frac{1}{2} \log R+C \quad(r \geqslant 1)
$$

then $\left\{e^{i \lambda_{n} t}\right\}$ is complete in $L^{2}[-\pi, \pi]$. This statement does not appear to be helpful in our situation. However, the proof of the theorem shows that if $f \in L^{2}[-\pi, \pi]$ and $\Lambda(r)$ is the number of real zeroes of $\hat{f}$ of modulus $\leqslant r$, and if $\Lambda$ satisfies the above condition, then $f=0$. The proof of this fact given in [4] remains valid if the zeroes of the entire function $\hat{f}$ are counted with multiplicities. Suppose $f \in$ $L^{2}\left(I_{K}\right)$ and $\left\langle f, t^{j} e^{i n t}\right\rangle=0$ for $n \in \mathbf{Z}, 0 \leqslant j \leqslant K$; we need to show that $f=0$. Let $g(t)=f((K+1) t)$, so that $g \in L^{2}(-\pi, \pi)$, and let $\Lambda(r)$ be the number of real zeroes of $\hat{g}$, of modulus $\leqslant r$. Now, $\hat{g}$ has a zero of order at least $K+1$ at each 
point $(K+1) n(n \in \mathbf{Z})$. Hence, if $[x]$ denotes the greatest integer $\leqslant x$, then

$$
\Lambda(r) \geqslant 2(K+1)[r /(K+1)]+K+1 .
$$

It follows that for any positive integer $n$ we have

$$
\begin{aligned}
& \int_{n(K+1)}^{(n+1)(K+1)} \frac{\Lambda(r)}{r} d r \geqslant \int_{n(K+1)}^{(n+1)(K+1)} \frac{(2 n+1)(K+1)}{r} d r \\
& \quad=(2 n+1)(K+1) \int_{0}^{(K+1) / 2}\left(\frac{1}{\left(n+\frac{1}{2}\right)(K+1)+t}+\frac{1}{\left(n+\frac{1}{2}\right)(K+1)-t}\right) d t \\
& \quad=(2 n+1)^{2}(K+1)^{2} \int_{0}^{(K+1) / 2} \frac{d t}{\left(n+\frac{1}{2}\right)^{2}(K+1)^{2}-t^{2}} \\
& \quad \geqslant(2 n+1)^{2}(K+1)^{2} \int_{0}^{(K+1) / 2} \frac{d t}{\left(n+\frac{1}{2}\right)^{2}(K+1)^{2}}=2(K+1) .
\end{aligned}
$$

This shows that for some constant $C$

$$
\int_{1}^{R} \frac{\Lambda(r)}{r} d r \geqslant 2 R+C \quad(R \geqslant 1)
$$

Thus the result of Levinson quoted above shows that $g=0$, and hence $f=0$; this finishes the proof of the completeness of the set $\left\{t^{j} e^{i n t}\right\}(n \in \mathbf{Z}, 0 \leqslant j \leqslant K)$ in $L^{2}\left(I_{K}\right)$.

Now, by Theorem 1, to show that this set is a Riesz basis we need only show that for any choice of scalars $c_{n, j}(n \in \mathbf{Z}, 0 \leqslant j \leqslant K)$ the system

$$
\left\langle f, t^{j} e^{i n t}\right\rangle=c_{n, j} \quad(n \in \mathbf{Z}, 0 \leqslant j \leqslant K)
$$

has a solution $f \in L^{2}\left(I_{K}\right)$ if and only if $\Sigma_{n j}\left|c_{n}\right|^{2}<\infty$.

Since $\left\langle f, t^{j} e^{i n t}\right\rangle=\left\langle t^{j} f, e^{i n t}\right\rangle$ and $t^{j} f \in L^{2}\left(I_{K}\right)$ if $f \in L^{2}\left(I_{K}\right)$, the necessity of the condition $\Sigma_{n j}\left|c_{n, j}\right|^{2}<\infty$ is evident. We show sufficiency by induction on $K$; the case $K=0$ is also perfectly standard.

For the induction step, suppose we have proved the theorem for $K=K_{0}$, and suppose that $\left\{c_{n, j}\right\}\left(n \in \mathbf{Z}, 0 \leqslant j \leqslant K_{0}+1\right)$ is a sequence of scalars with $\Sigma_{n, j}\left|c_{n, j}\right|^{2}$ $<\infty$; we need to find $f \in L^{2}\left(I_{K_{0}+1}\right)$ with

$$
\int_{I_{K_{0}+1}} f(t) t^{j} e^{-i n t} d t=c_{n j} \quad\left(n \in \mathbf{Z}, 0 \leqslant j \leqslant K_{0}+1\right) .
$$

By the induction hypothesis we can find $f_{1} \in L^{2}\left(I_{K_{0}}\right)$ with

$$
\int_{I_{K_{0}}} f(t) t^{j} e^{-i n t} d t=c_{n, j} \quad\left(n \in \mathbf{Z}, 0 \leqslant j \leqslant K_{0}\right) .
$$

Extend $f_{1}$ to be zero on $I_{K_{0}+1}-I_{K_{0}}$, so $f_{1} \in L^{2}\left(I_{K_{0}+1}\right)$. Now since $t^{K+1} f_{1}(t) \in$ $L^{2}\left(I_{K_{0}+1}\right)$ and the $c_{n j}$ are square summable we can find $g \in L^{2}\left(I_{0}\right)=L^{2}(-\pi, \pi)$ such that

$$
\int_{-\pi}^{\pi} g(t) e^{-i n t}=\left((i \pi)^{\left(K_{0}+1\right)}\left(K_{0}+1\right) !\right)^{-1}\left(c_{n, K_{0}+1}-\int_{I_{K_{0}+1}} f_{1}(t) t^{K_{0}+1} e^{-i n t} d t\right)
$$


By the proposition above we can find $f_{2} \in L^{2}\left(I_{K_{0}+1}\right)$ with $\hat{f}_{2}(z)=(\sin \pi z)^{K_{0}+1} \hat{g}(z)$ $(z \in \mathbf{C})$. Let $F=\hat{f}_{2}$; the derivatives of the entire function $F$ are given by

$$
F^{(j)}(z)=(2 \pi)^{-1 / 2}(-i)^{j} \int_{I_{K_{0}+1}} f_{2}(t) t^{j} e^{-i z t} d t
$$

But since $F(z)=(\sin \pi z)^{K_{0}+1} \hat{g}(z)$ we see that for $n \in \mathbf{Z}$

$$
F^{(j)}(n)=0 \quad \text { if } 0 \leqslant j \leqslant K_{0}, \quad F^{\left(K_{0}+1\right)}(n)=\pi^{\left(K_{0}+1\right)}\left(K_{0}+1\right) ! \hat{g}(n) .
$$

Combining (3) and (4) yields

$$
\int_{I_{K_{0}+1}} f_{2}(t) t^{j} e^{-i n t} d t=\left\{\begin{array}{l}
0 \quad\left(n \in \mathbf{Z}, 0 \leqslant j \leqslant K_{0}\right), \\
c_{n, K_{0}+1}-\int_{I_{K_{0}+1}} f_{1}(t) t^{K_{0}+1} e^{-i n t} \quad\left(n \in \mathbf{Z}, j=K_{0}+1\right) .
\end{array}\right.
$$

Hence (2) shows that if $f=f_{1}+f_{2}$, then $f$ is a solution to (1). Q.E.D.

LeMma 2. Let $\varphi_{n} \in C^{2}\left(I_{K+1}\right)(n \in \mathbf{Z})$, let $\varepsilon>0$, and suppose that $\left\|\varphi_{n}\right\|_{\infty} \leqslant \varepsilon$, $\left\|\varphi_{n}^{\prime}\right\|_{\infty} \leqslant \varepsilon$, and $\left\|\varphi_{n}^{\prime \prime}\right\|_{\infty}<\varepsilon$ for each $n \in \mathbf{Z}$. Then for any scalars $\left\{a_{n}\right\}$ and any $N$

$$
\int_{I_{K}}\left|\sum_{-N}^{N} a_{n} e^{i n t} \varphi_{n}(t)\right|^{2} d t \leqslant \varepsilon^{2} M^{2} \sum_{-N}^{N}\left|a_{n}\right|^{2},
$$

where $M$ depends only on $K$.

Proof. Fix $\psi \in C^{2}(\mathbf{R})$ with support $(\psi) \subseteq I_{K+1}, 0 \leqslant \psi \leqslant 1$, and $\psi(t)=1$ for $t \in I_{K}$. Let $f_{n}(t)=e^{i n t} \varphi_{n}(t) \psi(t)$; then

$$
\int_{I_{K+1}}\left|f_{n}(t)\right|^{2} d t \leqslant 2(K+2) \pi \varepsilon^{2}
$$

For $n \neq m$, let $F_{n, m}=\varphi_{n} \bar{\varphi}_{m} \psi^{2}$. Then

$$
F_{n, m}( \pm(K+1) \pi)=F_{n, m}^{\prime}( \pm(K+1) \pi)=0
$$

so integrating twice by parts gives

$$
\begin{aligned}
\left|\int_{I_{K+1}} f_{n} \bar{f}_{m} d t\right| & =\left|\int_{I_{K+1}} F_{n, m}(t) e^{i(n-m) t} d t\right| \\
& =\left|\int_{I_{K+1}} \frac{e^{i(n-m) t}}{(n-m)^{2}} F_{n, m}^{\prime \prime}(t) d t\right| \\
& \leqslant \frac{1}{(n-m)^{2}} \int_{I_{K+1}}\left|F_{n, m}^{\prime \prime}(t)\right| d t .
\end{aligned}
$$

By Leibniz' formula and the hypotheses on $\left\{\varphi_{n}\right\}$ we can certainly find $M^{\prime}$ (depending only on $K$ ) with

$$
\int_{I_{K+1}}\left|F_{n, m}^{\prime \prime}(t)\right| d t<M^{\prime} \varepsilon^{2} \quad(n, m \in \mathbf{Z}, n \neq m) .
$$


But now

$$
\begin{aligned}
\int_{I_{K}}\left|\sum_{-N}^{N} a_{n} e^{i n t} \varphi_{n}(t)\right|^{2} d t & \leqslant \int_{I_{K+1}}\left|\sum_{-N}^{N} a_{n} f_{n}\right|^{2} d t \\
& \left.=\left.\int_{I_{K+1}}\left|\sum_{-N}^{N}\right| a_{n}\right|^{2}\left|f_{n}\right|^{2}+\sum_{\substack{n, m=-N \\
n \neq m}}^{N} a_{n} \bar{a}_{m} f_{n} \bar{f}_{m}\right] d t \\
& \leqslant \sum_{-N}^{N}\left|a_{n}\right|^{2} \int_{I_{K+1}}\left|f_{n}\right|^{2} d t+\sum_{\substack{n, m=-N \\
n \neq m}}^{N}\left|a_{n} a_{m}\right|\left|\int_{I_{K+1}} f_{n} \bar{f}_{m} d t\right| \\
& \leqslant \varepsilon^{2} 2(K+2) \pi \sum_{-N}^{N}\left|a_{n}\right|^{2}+\varepsilon^{2} M^{\prime} \sum_{\substack{n, m=-N \\
n \neq m}}^{N} \frac{\left|a_{n} a_{m}\right|}{\left(n-m^{2}\right.} \\
& \leqslant \varepsilon^{2} 2(K+2) \pi \sum_{-N}^{N}\left|a_{n}\right|^{2}+\varepsilon^{2} M^{\prime} \sum_{\substack{m \neq 0 \\
m \neq 0}}\left(\frac{1}{m^{2}} \sum_{n}\left|a_{n+m} a_{n}\right|\right) \\
& \leqslant \varepsilon^{2} 2(K+2) \pi \sum_{-N}^{N}\left|a_{n}\right|^{2}+\varepsilon^{2} M^{\prime} \sum_{m \neq 0}\left(\frac{1}{m^{2}} \sum_{n=-N}^{N}\left|a_{n}\right|^{2}\right) \\
& =\varepsilon^{2}\left(2(K+2) \pi+\frac{M^{\prime} \pi^{2}}{3}\right) \sum_{n=-N}^{N}\left|a_{n}\right|^{2} \text { Q.E.D. }
\end{aligned}
$$

We now introduce notation for divided differences. If $f(z)$ is a function of a complex variable and $z_{0}, \ldots, z_{j}$ are distinct complex numbers, let $f\left[z_{0}\right]=f\left(z_{0}\right)$ and

$$
f\left[z_{0}, \ldots, z_{j}\right]=\frac{f\left[z_{0}, \ldots, z_{j-1}\right]-f\left[z_{1}, \ldots, z_{j}\right]}{z_{0}-z_{j}} .
$$

In order to fit exponentials into this scheme, we write $e^{i t z}=\exp _{t}(z)$.

In the theorem below $\left\{\omega_{n, j}\right\}$ will be a fixed set of complex numbers $(n \in \mathbf{Z}, 0 \leqslant j$ $\leqslant K$ ), such that $\omega_{n, j} \neq \omega_{n, k}$ if $j \neq k$. If $\left\{c_{n, j}\right\}$ is any set of complex numbers $(n \in \mathbf{Z}, 0 \leqslant j \leqslant K)$, write $c_{j}^{n}=c_{n, j}$, and

$$
c_{j, \ldots, k}^{n}=\frac{c_{j, \ldots, k-1}^{n}-c_{j+1, \ldots, k}^{n}}{\omega_{n, j}-\omega_{n, k}} \quad(n \in \mathbf{Z}, 0 \leqslant j<k \leqslant K) .
$$

We can now state

THEOREM 3. To each nonnegative integer $K$ there corresponds $\delta_{K}>0$ such that if $\left\{\omega_{n, j}\right\}(n \in \mathbf{Z}, 0 \leqslant j \leqslant K)$ is a set of distinct complex numbers with $\left|n-\omega_{n, j}\right|<\delta_{K}$ for $n \in \mathbf{Z}$ and $0 \leqslant j \leqslant K$, then the following conclusion holds:

The system

$$
\int_{I_{K}} f(t) e^{-i t \omega_{n j}} d t=c_{n, j} \quad(n \in \mathbf{Z}, 0 \leqslant j \leqslant K)
$$

has a solution $f \in L^{2}\left(I_{K}\right)$ if and only if

$$
\sum_{n=-\infty}^{\infty}\left(\left|c_{0}^{n}\right|^{2}+\left|c_{0,1}^{n}\right|^{2}+\cdots+\left|c_{0,1, \ldots, K}^{n}\right|^{2}\right)<\infty
$$

further, if (5) has a solution it is unique. 
Proof. Fix $K$. Clearly, given $f \in L^{2}\left(I_{K}\right)$ and scalars $\left\{c_{n, j}\right\}$, the statement (5) holds if and only if

$$
\int_{I_{K}} f(t) \exp _{t}\left[\omega_{n, 0}, \ldots, \omega_{n j}\right] d t=c_{0, \ldots, j}^{n} \quad(n \in \mathbf{Z}, 0 \leqslant j<K) .
$$

So, by Theorem 1, the conclusion of the present theorem is equivalent to the statement that the set

$$
\left\{\exp _{t}\left[\omega_{n, 0}\right], \exp _{t}\left[\omega_{n, 0}, \omega_{n, 1}\right], \ldots, \exp _{t}\left[\omega_{n, 0}, \ldots, \omega_{n, K}\right]\right\} \quad(n \in \mathbf{Z})
$$

(viewed as a set of functions of $t \in I_{K}$ ) forms a Riesz basis for $L^{2}\left(I_{K}\right)$. This will be proved as a consequence of Theorem 2 and Lemma 1, using Lemma 2.

For $z, \lambda \in \mathbf{C}$, let $\Phi(z,<)=e^{i z \lambda}$. Note that

$$
\left.\left(\frac{\partial}{\partial \lambda}\right)^{j} \Phi\right|_{(z, 0)}=(i z)^{j}
$$

Hence one sees, by a trivial refinement of the reasoning in [2, Theorem 2, p. 250], that the following statement holds (recall $K$ is fixed):

For any $\varepsilon>0$ there exists $\delta_{K}>0$ such that if $\lambda_{0}, \ldots, \lambda_{K}$ are distinct complex numbers with $\left|\lambda_{j}\right| \leqslant \delta_{K}(0 \leqslant j \leqslant K)$ then we have

$$
\left|(i z)^{j} / j !-\exp _{z}\left[\lambda_{0}, \ldots, \lambda_{j}\right]\right|<\varepsilon \quad(0 \leqslant j \leqslant K)
$$

for any $z \in \mathbf{C}$ with $|z| \leqslant(K+2) \pi+1$.

Note that, by Cauchy's estimates, the validity of (8) for $|z| \leqslant(K+2) \pi+1$ implies

$$
\left|\left(\frac{\partial}{\partial z}\right)^{l}\left(\frac{(i z)^{j}}{j !}-\exp _{z}\left[\lambda_{0}, \ldots, \lambda_{j}\right]\right)\right|<\varepsilon \quad(0 \leqslant j \leqslant K, l=0,1, \ldots)
$$

for all $z$ with $|z| \leqslant(K+2) \pi$. However,

$$
\frac{(i t)^{j}}{j !} e^{i n t}-\exp _{t}\left[\omega_{n, 0}, \ldots, \omega_{n, j}\right]=e^{i n t}\left(\frac{(i t)^{j}}{j !}-\exp _{t}\left[\lambda_{n, 0}, \ldots, \lambda_{n, j}\right]\right),
$$

where $n+\lambda_{n, j}=\omega_{n, j}$. Hence, by Lemma 2, we obtain the following:

For any $\varepsilon>0$ there exists $\delta_{K}>0$ such that if the $\omega_{n, j}$ are as in the statement of the theorem and $a_{-N}, \ldots, a_{N}$ are scalars, then

$$
\int_{I_{K}}\left|\sum_{-N}^{N} a_{n}\left(\frac{(i t)^{j}}{j !} e^{i n t}-\exp _{t}\left[\omega_{n, 0}, \ldots, \omega_{n, j}\right]\right)\right|^{2} d t \leqslant M^{2} \varepsilon^{2} \sum_{-N}^{N}\left|a_{n}\right|^{2} \quad(0 \leqslant j<K),
$$

where $M$ depends only on $K$. It follows from (9) that for any scalars $a_{n j}$ ( $n \in Z$, $0 \leqslant j \leqslant K)$

$$
\begin{gathered}
\int_{I_{K}}\left|\sum_{j=0}^{K} \sum_{n=-N}^{N} a_{n, j}\left(\frac{(i t)^{j}}{j !} e^{i n t}-\exp _{t}\left[\omega_{n, 0}, \ldots, \omega_{n, j}\right]\right)\right|^{2} d t \\
<2^{(K+1)} M^{2} \varepsilon^{2} \sum_{j=0}^{K} \sum_{n=-N}^{N}\left|a_{n j}\right|^{2} .
\end{gathered}
$$


Now, Theorem 2 shows that

$$
\left\{\frac{(i t)^{j}}{j !} e^{i n t}\right\} \quad(n \in \mathbf{Z}, 0 \leqslant j \leqslant K)
$$

forms a Riesz basis for $L^{2}\left(I_{K}\right)$, so (by Theorem 1) there exists $A_{K}>0$ so that for any scalars $a_{n, j}$

$$
A_{K}^{2} \sum_{j=0}^{K} \sum_{n=-N}^{N}\left|a_{n, j}\right|^{2} \leqslant \int_{I_{K}}\left|\sum_{j=0}^{K} \sum_{n=-N}^{N} a_{n, j} \frac{(i t)^{j}}{j !} e^{i n t}\right|^{2} d t .
$$

If $M$ is as in (9), pick $\varepsilon>0$ so that $2^{(K+1)} M^{2} \varepsilon^{2}<A_{K}^{2}$; now choose $\delta_{K}$ so that (9) holds with this $\varepsilon$, for $\left|n-\omega_{n j}\right|<\delta_{K}$; then (10) shows that the hypotheses of Lemma 1 are satisfied, so that the set (7) above is a Riesz basis for $L^{2}\left(I_{K}\right)$. Q.E.D.

The statement of Theorem 3 gives no information on the size of $\delta_{K}$ (although for any given $K$, a suitable value for $\delta_{K}$ could be obtained from a sufficiently careful examination of the proofs above, together with the proof of Theorem 2 on p. 250 of [2]). However, Theorem 3 has a corollary, the statement of which makes no mention of $\delta_{K}$ :

Corollary. Suppose $\left\{\omega_{n, j}\right\} \quad(n \in \mathbf{Z}, 0 \leqslant j \leqslant K)$ is a set of distinct complex numbers such that for each $j(0 \leqslant j \leqslant K)$ we have $\lim _{n \rightarrow \infty}\left|n-\omega_{n, j}\right|=0$. Then the conclusion of Theorem 3 holds. (I.e., the system (5) above has a solution $f \in L^{2}\left(I_{K}\right)$ if and only if (6) holds, and if (5) has a solution it is unique.)

Proof. As before, we need only show that the functions (7) (listed after the statement of Theorem 3 ) form a Riesz basis for $L^{2}\left(I_{K}\right)$, under the present hypothesis. Now, it follows easily from Theorem 1 that an independent set of vectors is a Riesz basis if it can be made into a Riesz basis by changing only finitely many terms. Thus, in view of Theorem 3, we need only show that the set (7) is independent. Hence it is enough to show that the set

$$
S=\left\{e^{i t \omega_{n_{j}}}\right\} \quad(n \in \mathbf{Z}, 0 \leqslant j \leqslant K)
$$

is independent, since a biorthogonal set for (7) could easily be obtained from one for $S$. Let $S^{\prime}=S-\left\{e^{i t \omega_{0,0}}\right\}$.

Theorem 3 shows that changing finitely many elements of $S^{\prime}$ results in a set which is not complete in $L^{2}\left(I_{K}\right)$. Hence, by considerations of codimension ("deficiency"), $S^{\prime}$ must be incomplete, as well. Now a result of Schwartz [7, p. 133] shows that an incomplete set of exponentials is independent; thus $S^{\prime}$ is independent. (Schwartz states his result only for $\omega_{n, j}$ real, but the proof applies to general $\omega_{n, j}$.) But this shows that $S$ is independent: Suppose not. Then, since $S^{\prime}$ is independent, $e^{i t \omega_{0,0}}$ must be in the closure of the linear span of $S^{\prime}$; hence the incompleteness of $S^{\prime}$ implies that $S$ is incomplete, whence the same theorem of Schwartz implies that $S$ is independent, a contradiction. Q.E.D.

\section{RefERENCES}

1. R. P. Boas, Jr., A general moment problem, Amer. J. Math. 63 (1941), 361-370.

2. E. Isaacsonand H. B. Keller, Analysis of numerical methods, Wiley, New York, 1966.

3. M. I. Kadec, The exact value of the Paley-Wiener constant, Soviet Math. Dokl. 5 (1964), 559-561. 
4. N. Levinson, Gap and density theorems, Amer. Math Soc. Colloq. Publ., Vol. 26, Amer. Math. Soc., Providence, R. I., 1940; reprinted 1963.

5. R. E. A. C. Paley and N. Wiener, Fourier transforms in the complex domain, Amer. Math. Soc., Providence, R. I., 1934; 3rd printing 1978.

6. F. Riesz and B. Sz.-Nagy, Functional analysis, Ungar, New York, 1955.

7. L. Schwartz, Approximation d'une fonction quelconque pardessommes d'exponentielles imaginaires, Ann. Fac. Sci. Univ. Toulouse (4) 6 (1943), 111-176.

8. R. M. Young, On perturbing bases of complex exponentials in $L^{2}[-\pi, \pi]$, Proc. Amer. Math. Soc. 53 (1975), 137-140.

Department of Mathematics, University of Wisconsin, Madison, Wisconsin 53706 